\title{
The use of orbitals and full spectra to identify misalignment
}

\author{
Michael Monte $^{\mathrm{a}}$, Florian Verbelen ${ }^{\mathrm{a}}$, Bram Vervisch ${ }^{\mathrm{a}, \mathrm{b}}$ \\ ${ }^{a}$ University of Ghent, Department of Industrial Systems and Product Design, Graaf Karel de \\ Goedelaan 5, B-8500, Kortrijk, Belgium \\ ${ }^{\mathrm{b}}$ University of Ghent, Department of Electrical Engineering, Systems and Automation, \\ Technologiepark 914, B-9052, Zwijnaarde, Belgium
}

\begin{abstract}
In this paper a 'SpectraQuest' demonstrator is used to introduce misalignment in a rotating set-up. The vibrations caused by misalignment are measured by two accelerometers on the bearings and two eddy-current probes on the shaft itself. A comparison is made between the classical spectral analysis, orbitals and full spectra. Orbitals are used to explain the physical interpretation of the vibration caused by misalignment. Full spectra allow to distinguish imbalance from misalignment by looking at the forward and reversed phenomena. This analysis is done for different kinds of misalignment, couplings and combined machinery faults.
\end{abstract}

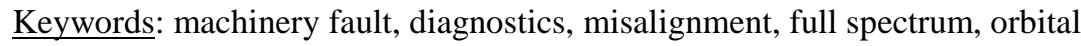

\section{Introduction}

In industry misalignment is one of the main causes of excessive vibrations leading to wear or even premature failure. The higher the speed, the bigger the impact of a small misalignment will be. The sooner misalignment is detected, the better for the condition of the machinery, energy consumption, and the costs of the maintenance of the set-up.

Several techniques, such as vibration monitoring, thermal imaging and oil particle analysis are used to detect and analyze machinery faults. Techniques to analyze the collected data is still an ongoing research topic. The most widespread method is the use of vibration spectra and that method will be used in this paper. The problem with the spectra is that it is not always obvious to distinguish between different types of faults such as misalignment or other machinery faults. To analyze the data, the time-based signal is measured by an accelerometer or eddy-current probe. From this signal the (half) spectrum is calculated in order to facilitate the analysis.

Modeling and analyzing simultaneous occurring faults in rotating machinery, is an ongoing research topic [1] [2]. New models and methods are still being developed and applied on test set-ups to find the best technique for every single fault and a combination of faults [3].

The type of misalignment has an impact on the spectrum. From the conducted measurements it is clear that the spectrum not only depends on parallel or angular misalignment, but also on the coupling, the excitation force and the rotating speed. Moreover, misalignment occurs generally in combination with other faults. Analysis of spectra when faults occur simultaneously demands experience and a thorough knowledge of the machinery.

Full spectra and orbitals need data from two orthogonal signals and are still being studied by different researchers [4], [5], [6], [7]. The vibration data can provide information about movement of the shaft and the direction of the vibration. The orbital has an elliptical shape due to the superposition of two circular movements. This forward and reverse movements are also shown in the frequency domain in a full spectrum and are a useful tool for analyzing machinery faults.

\section{Misalignment}

Misalignment is a well-known phenomenon and a lot of research is done in order to understand and detect this machinery fault. Several techniques exist to eliminate the different kinds of misalignment [8]. Alignment errors can be divided into four categories: horizontal offset, vertical offset, horizontal angle and vertical angle error. In reality misalignment consists of combinations of the above. A parallel misalignment occurs when the alignment error is limited to pure offset (horizontal or vertical). 
Parallel misalignment causes a shearing force and a bending moment at the end of the coupled shaft. This leads to a large radial reaction force in the bearings which is measured as vibration. This radial vibration is situated at the $1 \mathrm{x}, 2 \mathrm{x}$ and $3 \mathrm{x}$ order peaks in the spectrum. When the misalignment is very serious, the higher orders will also be excited. The peak at $2 \mathrm{x}$ can be partly explained as follows. When the shaft rotates there will be two excitations every rotation.

Angular misalignment will provide a bending moment resulting in a large axial vibration which can be measured at $1 \mathrm{x}, 2 \mathrm{x}$ and $3 \mathrm{x}$ order peaks. Unlike parallel misalignment the $1 \mathrm{x}$ component will be more pronounced, which makes it difficult to distinguish this error from imbalance.

\section{Experimental results}

The set-up used for the experiments is the Machinery Fault Simulator from SpectraQuest. Besides using the simulator as an educational tool it can also be used for research [9]. For the measurements two sensors are needed. For these measurements accelerometers or eddy-current probes were used. Before discussing the different faults, reference measurements had to be made in order to see the difference between a good aligned set-up and a misaligned set-up. Fig. 1 shows the spectrum of data from an accelerometer. Peaks are expected at $1 \mathrm{x}(35 \mathrm{~Hz})$ (little imbalance) and 2x (70 Hz) (small misalignment). The presence of a peak at $3 \mathrm{x}(105 \mathrm{~Hz})$ is due to the flexible coupling. This coupling makes the $2 \mathrm{x}$ peak more pronounced. The peaks $1 \mathrm{x}$ and $2 \mathrm{x}$ at the second lager are also bigger compared to the first lager (closer to the coupling). The displacement at the lager will become bigger the further it is from the coupling.
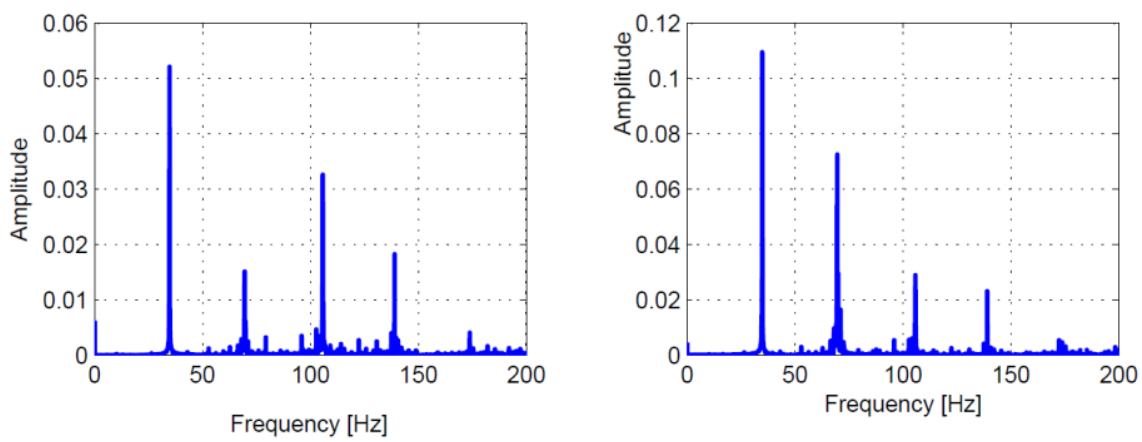

Fig. 1 Horizontal measurement without imbalance

First measurements on parallel misalignment are treated, then angular misalignment is discussed. The measurements on parallel and angular misalignment have been carried out at different rotational speeds. For parallel misalignment a flexible coupling had to be used. The rigid coupling does not allow parallel misalignment. For the angular misalignment both the flexible and the rigid coupling are used to show the difference between the two couplings. The sample frequency was 2048 $\mathrm{Hz}$ and the measuring time was $10 \mathrm{~s}$ for all measurements.

\section{a. Measurements on parallel misalignment}

With parallel misalignment a peak is expected at $2 \mathrm{x}$ the rotational speed in the spectrum of the signal. This peak has a greater amplitude compared to the $1 \mathrm{x}$ peak. This is according to the characteristics of parallel misalignment. This peak is shown in the horizontal (Fig. 2) and vertical (Fig. 3) (half) spectrum. The large peak at $3 \mathrm{x}$ the rotational speed $(3 \mathrm{x} 35 \mathrm{~Hz})$ comes from the flexible coupling used in the set-up.

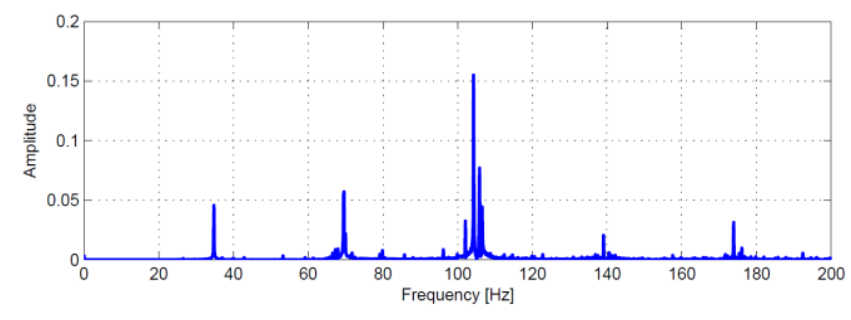

Fig. 2 Horizontal measurement on parallel misalignment 


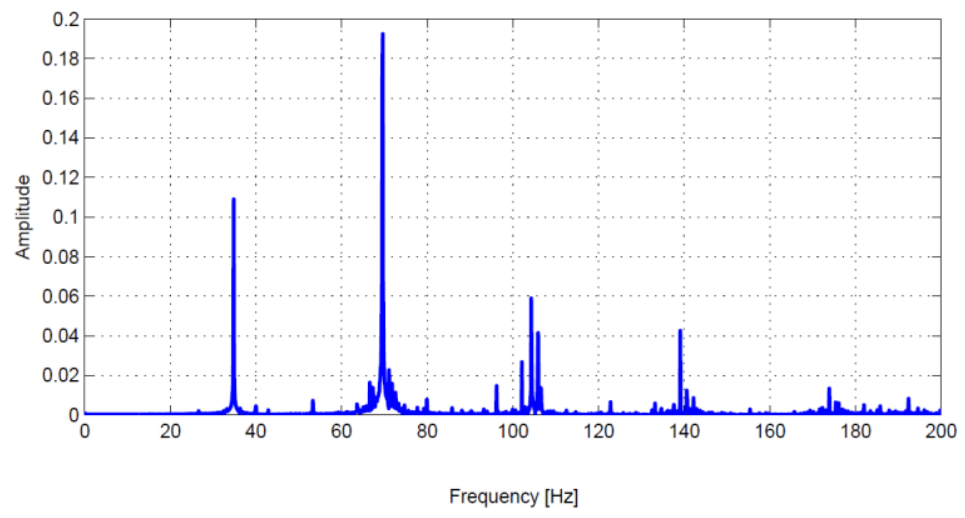

Fig. 3 Vertical measurement on parallel misalignment

The movement of the shaft under the influence of parallel misalignment is now studied with orbitals. In Fig. 4 measurements with an accelerometer are shown and the movement of the $1 \mathrm{x}$ vibration is plotted together with the $2 \mathrm{x}$ vibration. These plots are ellipses and they do not show any distortion. The third plot is the movement of the $1 \mathrm{x}$ and $2 \mathrm{x}$ vibration together. This figure shows that the movement of the shaft is disturbed. This is clearly a sign of parallel misalignment. The same results are obtained when measuring with an eddy-current probe (Fig. 5).
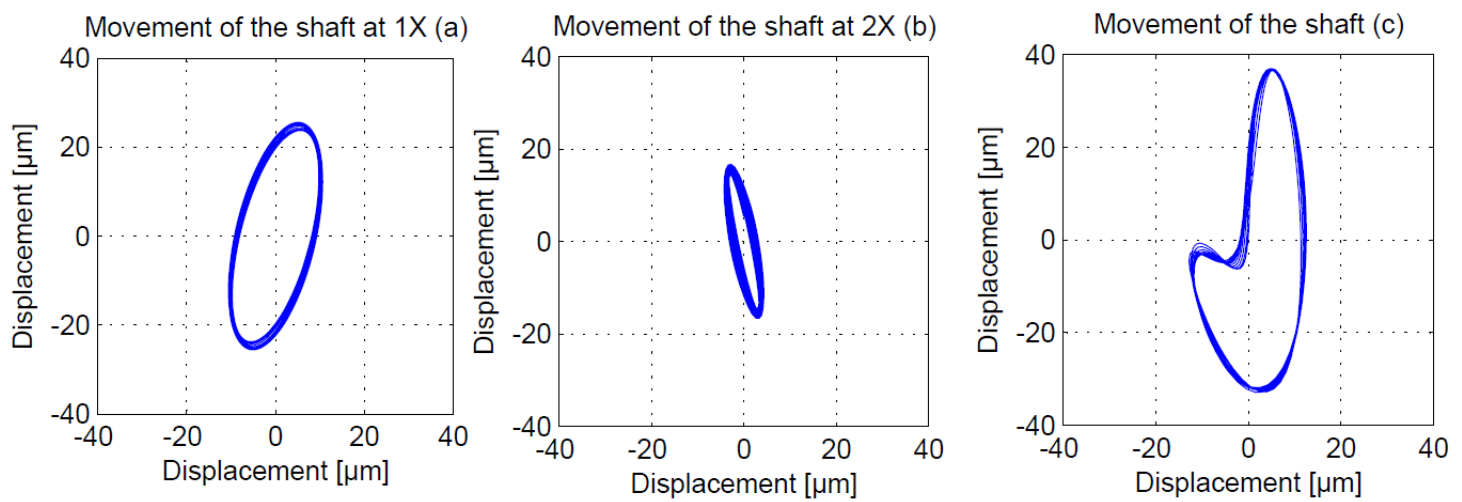

Fig. 4 Movement of the rotor as a result of parallel misalignment (accelerometer)
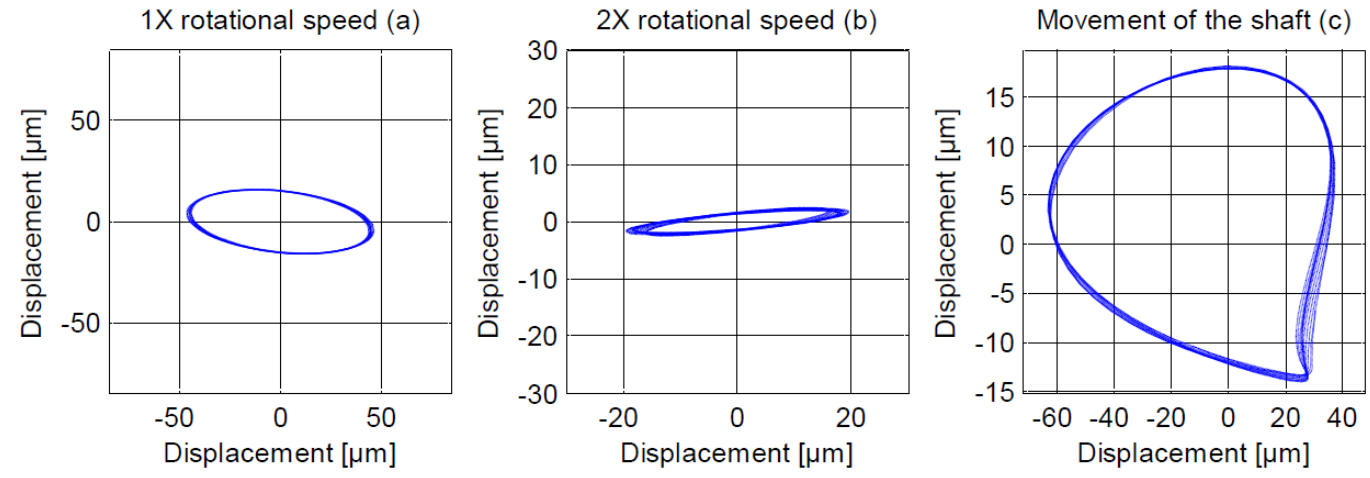

Fig. 5 Movement of the rotor as a result of parallel misalignment (eddy-current probe)

To study the influence of imbalance the measurements are done again with static imbalance added to the setup. The influence of the imbalance is shown by the enlargement of the orbital at $1 \mathrm{x}$ the rotational speed while the shape remains the same. The influence of the $2 \mathrm{x}$ vibration is decreased and shown in the orbital at 2x which indicates parallel misalignment (Fig. 6). 

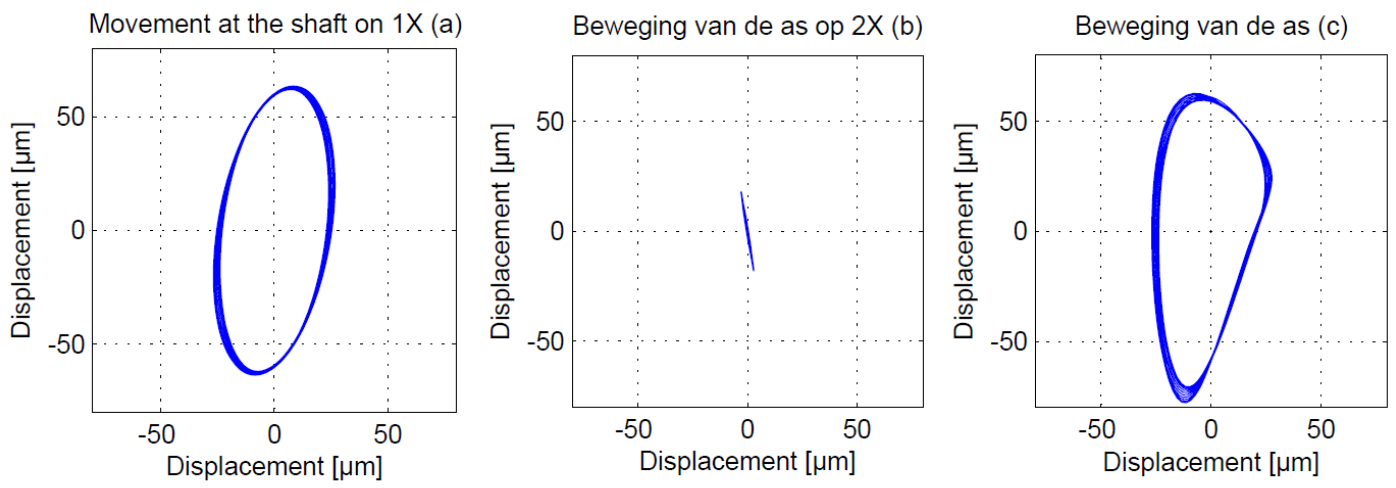

Fig. 6 Movement of the rotor as a result of parallel misalignment and imbalance

\section{b. Measurements on angular misalignment}

For angular misalignment a big 1x component is expected (Fig. 7 and Fig. 8). The problem with these spectra is that they resemble imbalance problems. Wrong conclusions can be drawn very easily.

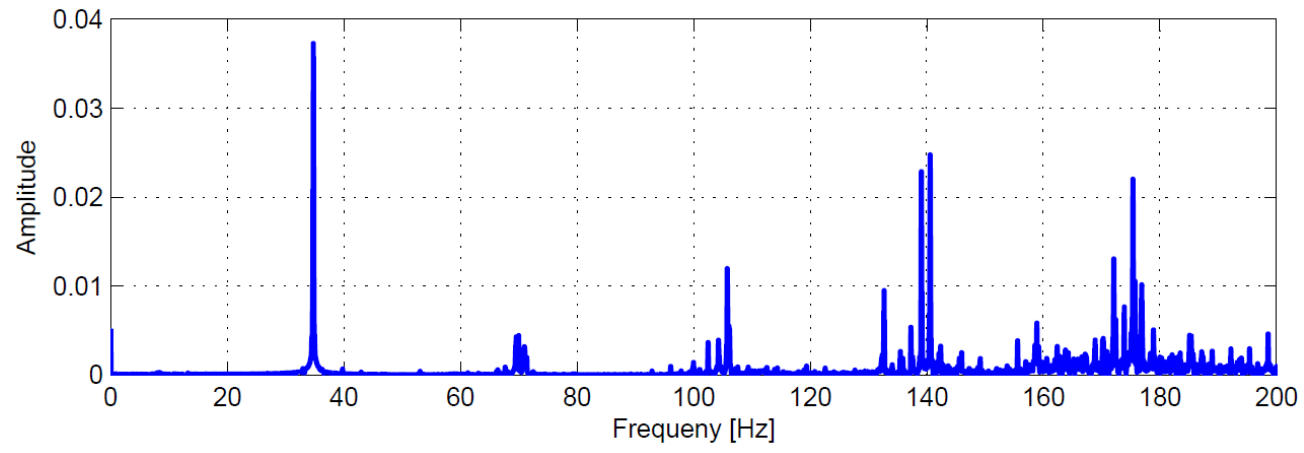

Fig. 7 Horizontal measurement on angular misalignment

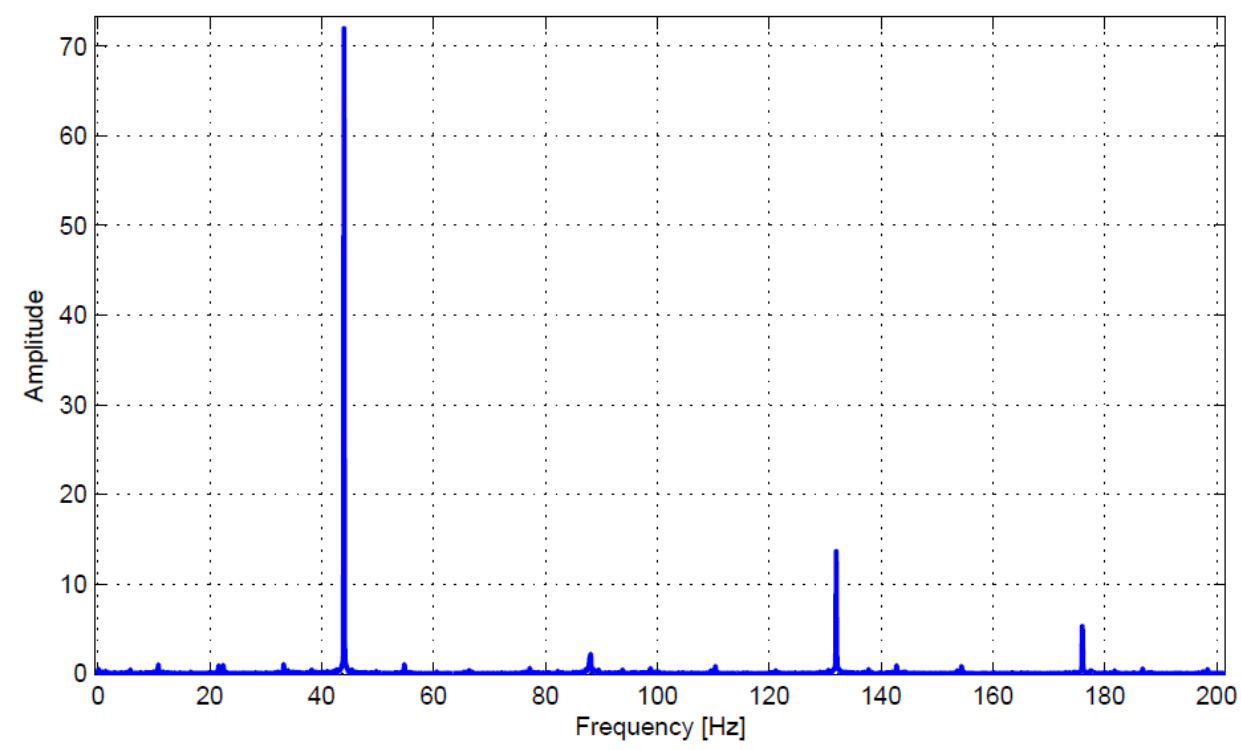

Fig. 8 Horizontal measurement on angular misalignment $(45 \mathrm{~Hz})$ 
Orbitals offer an excellent solution because the movement of the shaft when it is in imbalance is different compared to angular misalignment. Fig. 9 shows the orbitals of the $1 x, 2 x$ and a total image of the shaft movement measured by an accelerometer. From the $1 \mathrm{x}$ component no conclusion can be drawn. This again is an ellipse. On the other hand, the $2 \mathrm{x}$ component exhibits a very special form. Whereas the $2 \mathrm{x}$ component in an imbalance problem leads to an ellipse, the figure is distorted. This image is typical for angular misalignment with a flexible coupling. The figure shows the difference with imbalance problems. The same image is seen when looking at the orbitals from measurements by eddy-current probes (Fig. 10). However, the preliminary alignment was not perfect. A combination of a parallel and angular misalignment is present.
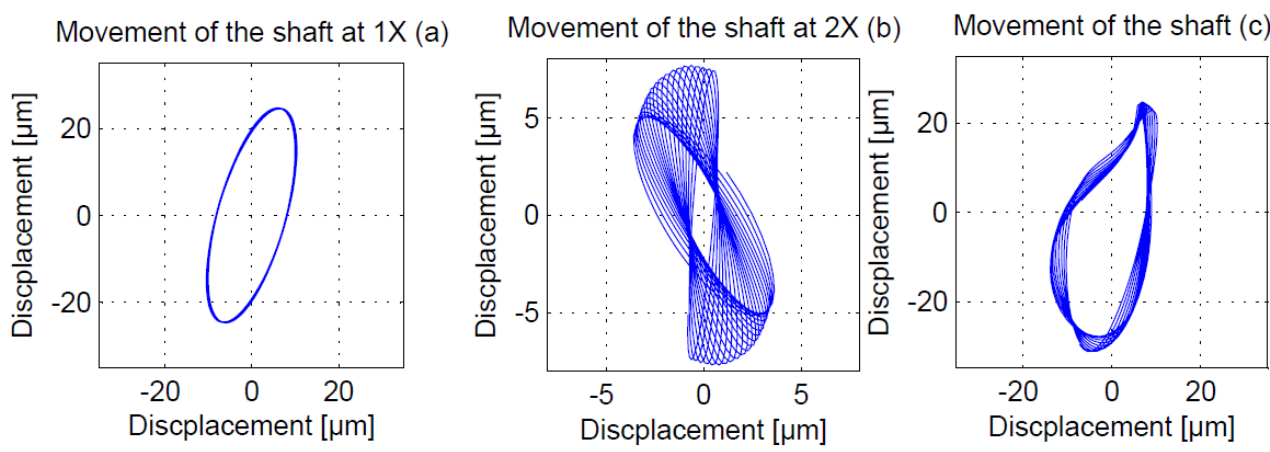

Fig. 9 Movement of the rotor as a result of angular misalignment with a flexible coupling
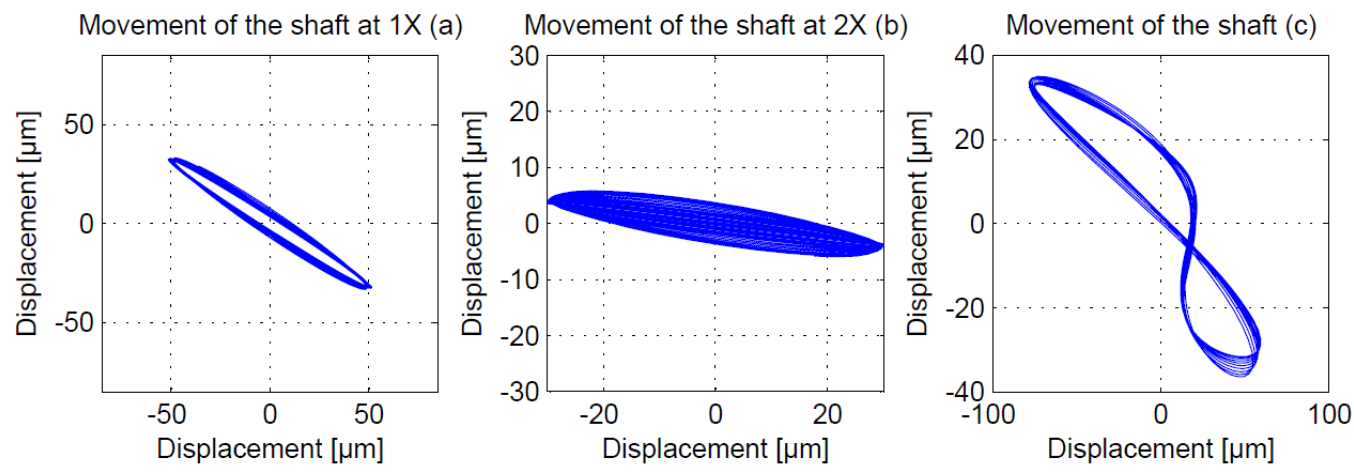

Fig. 10 Movement of the rotor as a result of angular misalignment with a flexible coupling (eddy-current probe)

In Fig. 11 the orbital of angular misalignment with a rigid coupling is shown. Note that the $2 \mathrm{x}$ image again is an ellipse in contrast to the flexible coupling. The rigid coupling does not allow the same movement as a flexible coupling.
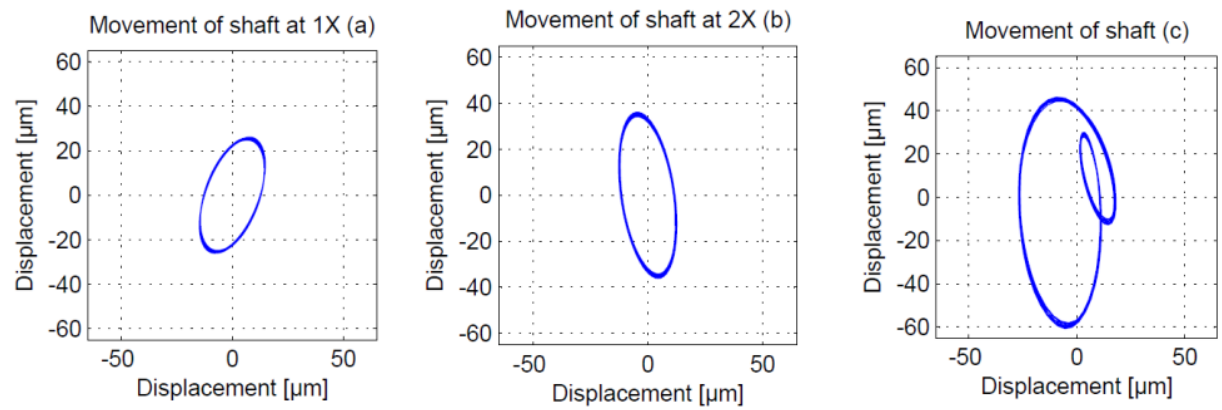

Fig. 11 Movement of the rotor as a result of angular misalignment with a rigid coupling

\section{c. Measurements on combined faults}

Many combined faults can lead to misinterpretation. In the spectrum of Fig. 12 a fault is shown that can be interpreted as an imbalance problem, an angular misalignment or a parallel misalignment with imbalance. Just as in the previous cases an 
orbital can give clarification. An alternative tool to detect the difference between imbalance and misalignment is the full spectrum (Fig. 13). The difference between the positive and negative frequencies gives a clarification.

Fig. 13 shows the full spectrum of Fig. 12. The peak at $1 \mathrm{x}$ is clearly larger than the $-1 \mathrm{x}$ peak which indicates imbalance. There is also a clear peak on both $2 \mathrm{x}$ as $-2 \mathrm{x}$ which indicates parallel misalignment. The problem shown is parallel misalignment with imbalance. From the original spectrum it is difficult to come to the same conclusion. The full spectrum, on the other hand, makes it fairly easy to draw a conclusion. A similar result is shown in Fig. 14 with measurements with an eddy-current probe.

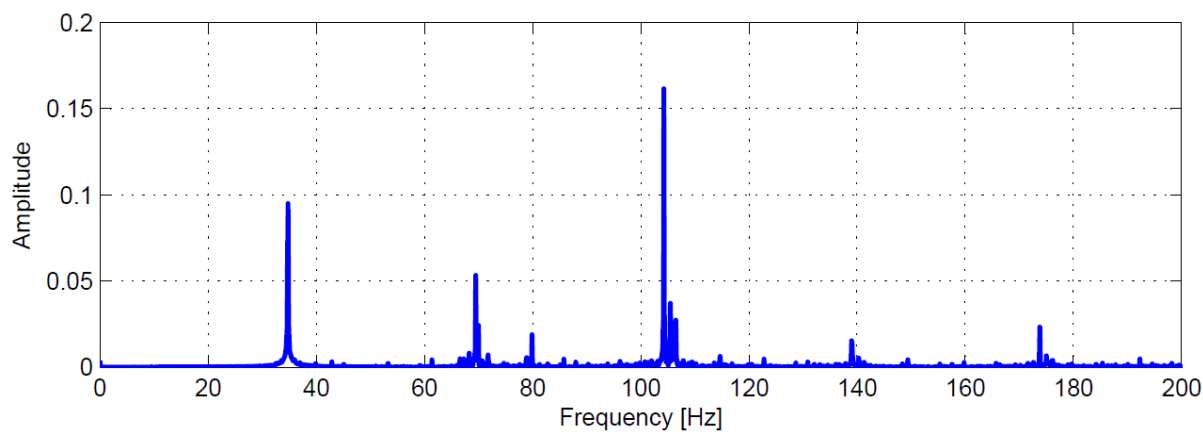

Fig. 12 (half) spectrum of parallel misalignment with imbalance

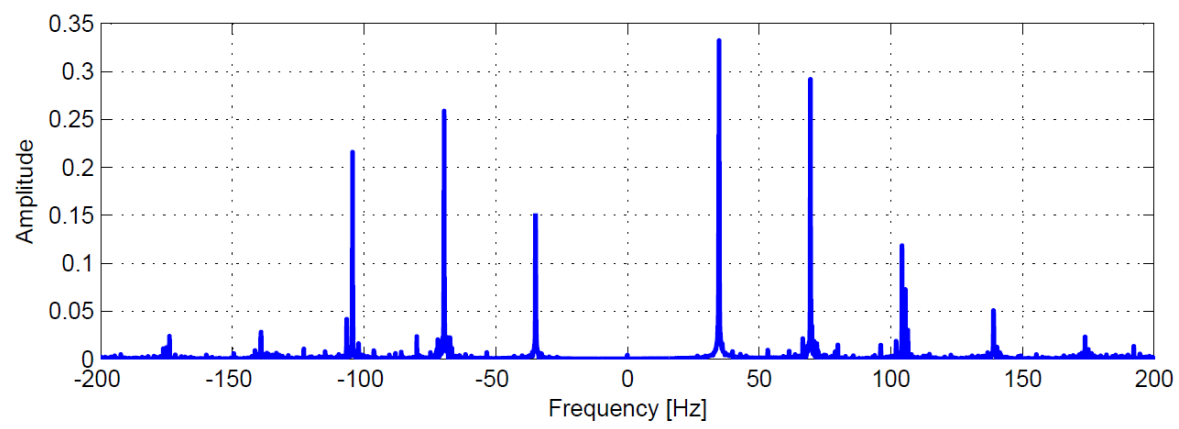

Fig. 13 Full spectrum of parallel misalignment with imbalance

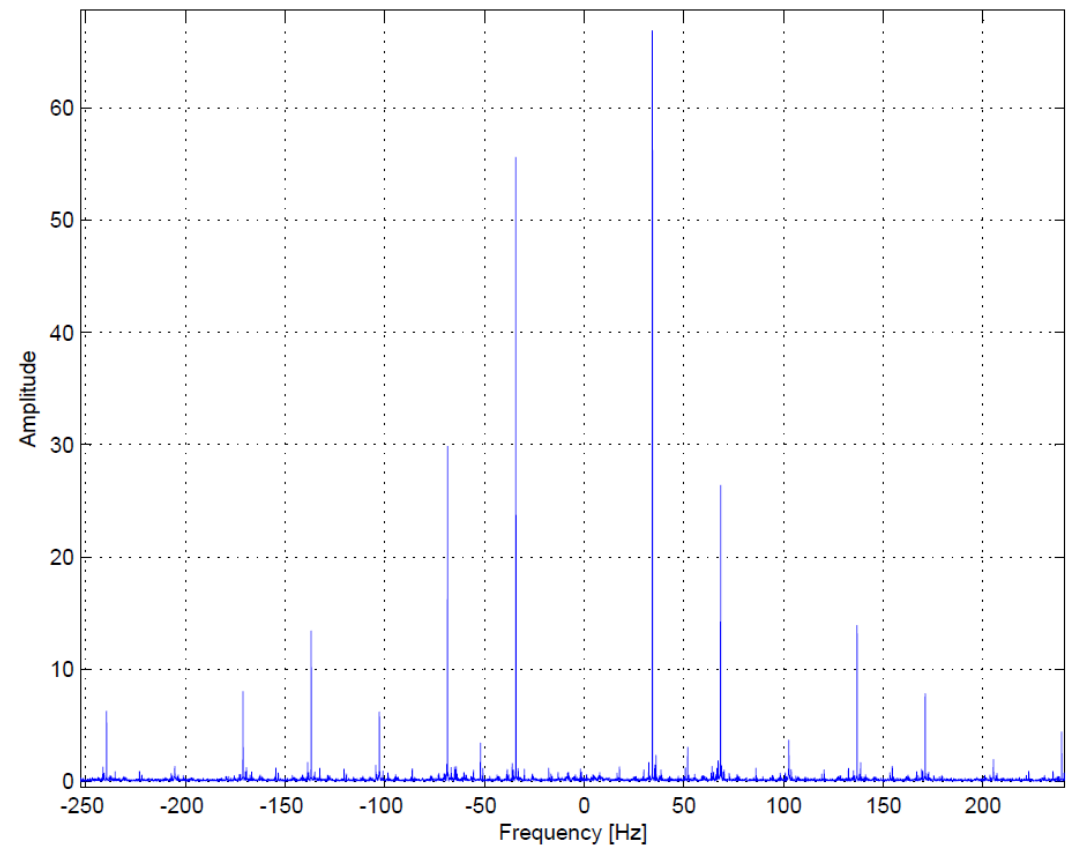

Fig. 14 Full spectrum of parallel misalignment with imbalance (eddy-current probe) 


\section{Conclusions}

Orbitals and full spectra have proven their usefulness in detecting machinery faults from vibration measurements. Misalignment is not always as clear to detect compared to imbalance in a (half) spectrum. For misalignment orbitals and full spectra can be used to make a proper analysis of the problem. An important finding from the many measurements is that the difference between parallel and angular misalignment can be seen in orbitals by the presence or absence of a twist in the contour. The presence of the twist indicates angular misalignment.

Imbalance mainly generates a forward vibration meaning that imbalance influences only the positive frequency range. Misalignment is evenly represented in the positive and negative frequency range. Summarized combined fault leads to the following in full spectra. For imbalance a large 1x peak and a small -1x peak is expected while for angular misalignment an equally large $1 \mathrm{x}$ and $-1 \mathrm{x}$ peak is expected. For parallel misalignment combined with imbalance is a combination expected.

The coupling also plays an important role in the measured spectrum and the shape of the resulting orbitals. Besides the large $1 \mathrm{x}$ and $2 \mathrm{x}$ vibration peaks the $3 \mathrm{x}$ component is also used.

Additional research is absolutely needed in order to confirm these findings. Furthermore, the different faults in a system and their influence on each other needs to be studied. Also the conclusion about the coupling is an interesting research topic. Nevertheless, at present the orbitals and full spectrum can be used as an additional tool when vibrational data are studied.

\section{References}

[1] N. Bachschmid, P. Pennacchi, and A. Vania, "Identification of multiple faults in rotor systems," J. Sound Vib., vol. 254, pp. 327-366, 2002.

[2] M. Lal and R. Tiwari, "Multi-fault identification in simple rotor-bearing-coupling systems based on forced response measurements," Mech. Mach. Theory, vol. 51, pp. 87-109, 2012.

[3] J. K. Sinha and K. Elbhbah, "A future possibility of vibration based condition monitoring of rotating machines," Mech. Syst. Signal Process., vol. 34, no. 1-2, pp. 231-240, Jan. 2013.

[4] P. Goldman and A. Muszynska, "Application of full spectrum to rotating machinery diagnostics," Orbit, pp. 17-21, 1999.

[5] F. Balitsky and A. Sokolova, "Application of Full Spectrums and other Vibration Two-dimensional Characteristics for Gas Transportation Units Condition Monitoring," in 10th European Conference on Non-Destructive Testing, 2010, no. 1, pp. 2-6.

[6] D. Southwick, "Using full spectrum plots,” Orbit, vol. 15, no. 2, pp. 11-15, 1994.

[7] J. Tůma and J. Biloš, "Fluid induced instability of rotor systems with journal bearings," Eng. Mech., vol. 14, no. 1, pp. 69-80, 2007.

[8] J. Piotrowski, Shaft alignment handbook. 2010.

[9] S. Ganeriwala, S. Patel, and H. A. Hartung, "The truth behind misalignment vibration spectra of rotating machinery," Proc. Intl. Modal Anal. ..., pp. 2078-2085, 1999. 